\title{
Exosomes Derived from Dendritic Cells Attenuate Liver Injury by Modulating the Balance of Treg and Th17 Cells After Ischemia Reperfusion
}

\author{
Lei Zheng Zhi Li Wei Ling Deming Zhu Zhiwen Feng Lianbao Kong \\ Liver Transplantation Center, The First Affiliated Hospital of Nanjing Medical University, Nanjing, China
}

\section{Key Words}

Dendritic cells • Exosome • Tregs • Th17 • Liver ischemia reperfusion injury

\begin{abstract}
Background/Aims: The present study aimed to evaluate the effects as well as the underlying mechanisms of bone marrow-derived dendritic cells (BMDCs) and exosomes produced by BMDCs (DEXs) on hepatic ischemia-reperfusion (I/R) injury (IRI). Methods: Primary hepatocytes were isolated and used to mimic the liver IR microenvironment. BMDCs were induced and characterized both biochemically with a flow cytometer (FCM) and biophysically with a microscope. Then, we exposed BMDCs to the supernatants from primary hepatocytes and evaluated the maturation of BMDCs by FCM. BMDCs were systemically injected into mice before liver IR via the tail vein, and the therapeutic effects were evaluated. The serum levels of transaminases (aspartate aminotransferase (AST) and alanine aminotransferase (ALT), inflammatory cytokines, and histological changes were respectively examined by ELISA, RTqPCR and microscopy. Furthermore, we isolated DEXs by ultracentrifugation, characterized DEXs by transmission electron microscopy (TEM) and nanosight tracking analysis (NTA) and western blotting (WB), and then we co-cultured BMDCs/DEXs and naïve T cells and performed FCM, ELISA and confocal imaging. Moreover, we injected DEXs into mice prior to liver IR via the tail vein and examined its therapeutic effects by microscopy and ELISA. Finally, inhibitors of HSP70 (cmHSP70.1), PI3K (BKM120) and mTOR (Rapamycin) were used to investigate the role of HSP70 and the PI3K/mTOR axis in the effects of DEXs on naïve T cells by WB and FCM. Results: Bone marrow cells were efficiently induced into dendritic cells (DCs) with typical DC characteristics. The supernatants from primary hepatocytes exposed to H/R upregulated DC maturation markers. After DC administration, liver IR injury was improved with histopathological scores and serum transaminases. Additionally, we found that the anti-inflammatory cytokines TGF- $\beta$, Foxp3 and interleukin (IL)-10 were upregulated and that IL-17 was downregulated. Furthermore, confocal imaging revealed that the uptake of H/R-DEXs by naïve $T$ cells was greater than that of DEXs derived from the control or negative group of BMDCs, and this
\end{abstract}


Zheng et al.: Dexs Attenuate Liver Ischemia Reperfusion Injury by Modulating the Balance of Treg and Th17

increase was correlated with a significantly greater degree of differentiation of Tregs and Th17 cells. Moreover, H/R-DEXs administration improved liver function in mice after IR. Finally, the inhibition of HSP70, PI3K and mTOR completely abolished the effect of DEXs on naïve T cells. Conclusion: These results demonstrated that BMDCs and DEXs could alleviate hepatic I/R injury via modulating the balance between Tregs and Th17 cells. DEXs transported HSP70 into naïve T cells and stimulated the PI3K/mTOR axis to modulate the balance between Tregs and Th17 cells and protect the liver from IR injury.

(C) 2018 The Author(s)

Published by S. Karger AG, Basel

\section{Introduction}

Hepatic ischemia and reperfusion (I/R), an important clinical problem that affects hepatic surgeries and liver transplantation, frequently induces the injury of remote organs, including the kidney, lung, and heart. In particular, acute kidney injury (AKI) after liver I/R is common (40-85\% incidence) and greatly increases patient mortality and morbidity during the perioperative period [1-5]. Therefore, reducing the adverse effects of I/R injury (IRI) will increase the lifespan of patients after hepatic surgeries.

Innate and adaptive immune responses play important roles in liver IRI. Recent studies have confirmed that dendritic cells (DCs) play a key role in the pathogenesis of liver IRI. Using warm and cold hepatic I/R models, studies have shown different roles for liver-resident versus blood-borne DCs, highlighting the importance of the local microenvironment in determining DC function during hepatic IRI [6-8]. Additionally, a previous study determined that CD4+ T cells play an important role in liver IRI [9]. Activated CD4+ T cells exhibit various immune functions with distinctive phenotypes. Research has determined that cytokine secretions are the primary effector mechanism in differentiating these CD4+ T cell subsets. CD4+ T cells include not only Th1 and Th2 cells but also T helper 17 cells (Th17), Tregs and $\mathrm{T}$ follicular helper cells (Tfh). Previous studies have also shown that RAR-related orphan receptor gamma $(\mathrm{ROR} \gamma)+\mathrm{IL}-17+$ neutrophils and IL-17A-producing natural killer cells play critical roles in a modified model of liver IRI, which occurred in RAG-deficient hosts [10]. Another study demonstrated that ex vivo-induced Treg cells could protect livers from IRI by inhibiting the activation of Kupffer cells in a liver partial warm ischemia model [11]. Moreover, some studies have demonstrated that specific $\mathrm{T}$ cell populations, such as effector memory CD4+ T cells, play key roles in the I/R process by secreting cytokines and expressing costimulatory molecules to either promote or inhibit innate immune activation or facilitate tissue repair/homeostasis. Additionally, subsets of CD4+ T cells, such as regulatory T cells (Tregs) and Th17 cells, play important roles in liver IRI [12]. Thus, the relationship among DCs, Tregs and Th17 cells plays a key role in liver IRI and could potentially be used as a therapeutic target.

In recent studies, a novel mode of intercellular communication mediated by exosomes, a class of extracellular vesicles, has been implicated in regulating inflammation [13, 14]. Exosomes are small double-membrane vesicles secreted via exocytosis from DCs, macrophages, T cells and cells of other tissue origins under physiological and pathological conditions. Furthermore, the exosomes from antigen-presenting cells can confer therapeutic benefits by attenuating or stimulating the immune response [15]. A recent study showed that dendritic cell-derived exosomes (DEXs) deliver antigen-specific signals and can be regarded as inert vehicles that mimic DCs and activate T cells [16]. The biological function of DEXs in tumors and inflammatory diseases has been demonstrated in a number of studies [17-19]. However, to our knowledge, there have been no studies investigating the biological function of DEXs in liver I/R or whether DEXs can modulate CD4+ T cell activation and differentiation.

Here, we investigated the hepatoprotective effect as well as the underlying mechanisms of BMDCs and DEXs on hepatic I/R injury. We demonstrated that DEXs may act as activators of CD4+ T cells, which improve liver IRI. Based on these findings, we report a novel endocrine DEX-mediated communication mechanism between DCs and CD4+ T cells that regulates the differentiation of CD4+ T cells and attenuates liver IRI. 


\section{Materials and Methods}

\section{Study design}

Fig. 1 shows an outline of the present study. Briefly, we used C57B/L mice as donors for the culture of hepatocytes, bone marrow-derived DCs (BMDCs) and naïve T cells and as the model of liver I/R. Supernatants from primary hepatocytes exposed to hypoxia and reoxygenation $(\mathrm{H} / \mathrm{R})$ were applied to mimic the liver I/R microenvironment. We examined the uptake of DEXs by naïve T cells using confocal microscopy. Enzyme-linked immunosorbent assays (ELISA) were used to detect cytokine and protein expression. A flow cytometer was employed to monitor naïve $\mathrm{T}$ cell differentiation and proliferation.

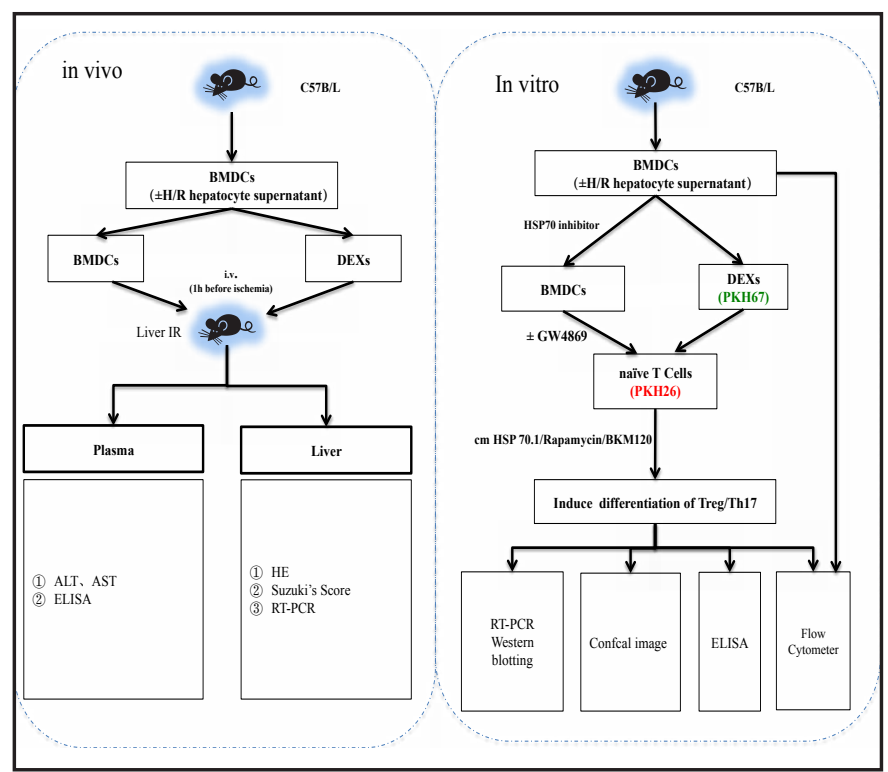

Fig. 1. Study design. Real-time quantitative PCR (RT-qPCR) and WB were performed to detect the expression of mRNA and protein, respectively. Using morphometric analysis and immunohistochemistry, liver IRI and the differentiation of Tregs and Th17 cells were evaluated in vivo.

\section{Mice}

Male wild type 6-8-week-old mice (C57BL/6) were purchased from The Model Research Center Of Nanjing University and housed under pathogen-free conditions. We used 6- and 8-week-old mice as donors for the culture of BMDCs and naïve T cells, respectively. We injected DEXs into 8-week-old mice, which served as liver IRI models. Animal care and treatment complied with standards approved by the Institutional Review Board of Nanjing Medical University.

\section{Induction of $I / R$ and injection of BMDCS/DEXS}

We utilized the established non-lethal model of segmental (70\%) hepatic warm ischemia/reperfusion as previously described [20]. Briefly, we anesthetized 8-week-old C57BL mice by ether inhalation and then interrupted the artery and portal venous blood supply to the left and middle liver lobes for 60 min. Body temperature was maintained at $37^{\circ} \mathrm{C}$ using a warming pad and heat lamp. At the end of the predetermined period following reperfusion, the mice were anesthetized via ether inhalation and euthanized by cervical dislocation for tissue and plasma collection. We injected $1 \times 10^{6}$ BMDCs or $10 \mu \mathrm{g}$ of DEXs suspended in $100 \mu \mathrm{l}$ of PBS into the recipient mouse through the tail vein at $1 \mathrm{~h}$ prior to I/ $\mathrm{R}(\mathrm{n}=6$ per group). There was not any reaction at the injection site.

\section{Morphometric analysis}

To analyze the size and morphology of ischemia/reperfusion area, the liver tissue was fixed in paraformaldehyde and subsequently embedded in paraffin. Then, 5 - $\mu$ m-thick sections were prepared and stained with hematoxylin and eosin. Finally, the tissue sections were examined using light microscopy and evaluated by Suzuki's score [21].

\section{BMDC culture}

We isolated BMDCs from male 6-week-old C57B/L mice $(n=6)$ as previously described [22]. Briefly, bone marrow was isolated from male 6-week-old mice, the RBCs were lysed, and the sample was washed three times with PBS. We cultured the BMDCs at $10^{6}$ cells $/ \mathrm{ml}$ in Petri dishes (Corning, New York, USA) in complete RPMI-1640 medium (Gibco, California, USA) containing 10\% FBS (Gibco, California, USA) and $20 \mathrm{ng} / \mathrm{ml}$ recombinant granulocyte-macrophage colony-stimulating factor (PeproTech, New Jersey, USA). 


\section{Cellular Physiology Cell Physiol Biochem 2018;46:740-756 \\ \begin{tabular}{l|l} 
and Biochemistry Published online: April 05, 2018 & $\begin{array}{l}\text { (c) } 2018 \text { The Author(s). Published by S. Karger AG, Basel } \\
\text { www.karger.com/cpb }\end{array}$
\end{tabular}}

Zheng et al.: Dexs Attenuate Liver Ischemia Reperfusion Injury by Modulating the Balance of Treg and Th17

FBS-derived exosomes were depleted by overnight ultracentrifugation. Another $10 \mathrm{ml}$ of complete medium was added after 3 days. Following a routine culture protocol, the medium was changed every half day, and cytokinesis added to maintain the concentration of GM-CSF. On day 10, the BMDC cells were observed by inverted microscope, stained with anti-CD11c-PerCP/Cy5 and analyzed using a FACSCanto flow cytometer (BD, New York, USA).

\section{Isolation and culture of primary hepatocytes}

Six-week-old male C57B/L mice $(n=6)$ were used to isolate and culture primary hepatocytes according to a previously described protocol [23]. Briefly, we used ether to perform inhalation anesthesia. Subsequently, we cannulated the infrarenal IVC and transected the portal vein to enable adequate drainage of blood and perfusion media. We perfused the liver with approximately $30 \mathrm{ml}$ of liver perfusion media (Gibco, California, USA) and then switched to liver digestion media (Life Technologies, California, USA). In a cell culture hood, we transferred the liver from a 50-mL polypropylene conical tube containing digestion media into a sterile tissue culture dish and removed undigested tissue particles and cell debris. The cells were subsequently plated in $2 \mathrm{~mL}$ of Williams' Media E 1X (Gibco, California, USA) containing supplements (Life Technologies, California, USA) for thawing and plating primary hepatocytes on collagen-coated six-well plates. After $4 \mathrm{~h}$, the medium was changed to Williams' Media E 1X containing supplements (Life Technologies, California, USA) for the maintenance of primary hepatocytes.

\section{Simulation of the liver IR hepatocyte microenvironment}

We used the supernatants from primary hepatocytes exposed to H/R as previously described [24] to mimic the liver I/R microenvironment.

To generate H/R conditions, we washed the primary hepatocytes three times with PBS and used the AnaeroPack-Anaero Anaerobic Gas Generator (MGC, Tokyo, Japan) to absorb $\mathrm{O}_{2}$ and produce $\mathrm{CO}_{2}$. Following the manufacturer's instructions, a pink oxygen indicator that turned blue when the $\mathrm{O}_{2}$ concentration was greater than $5 \%$ was used to detect $\mathrm{O}_{2}$. We placed the culture plate in an incubator with $5 \% \mathrm{CO}_{2} / 95 \% \mathrm{~N}_{2}$ under constant temperature and humidity for $3 \mathrm{~h}$. The plate was subsequently removed from the hypoxia chamber and placed in a normoxic environment for $6 \mathrm{~h}$. Serum-free medium was applied to mimic the ischemic condition. At the conclusion of the reoxygenation period, we collected the supernatants to stimulate BMDCs.

In addition, we removed cell membrane particles by centrifugation at $1500 \mathrm{~g}$ for $30 \mathrm{~min}$ and collected $100 \mu \mathrm{l}$ of the supernatant. We added the supernatants to $1 \times 10^{6} \mathrm{BMDCs}$, followed by culture for $24 \mathrm{~h}$. We used the same conditions with the supernatants from normal hepatocytes (Control group) and the supernatants from the culture of BMDCs (Negative group). After $24 \mathrm{~h}$, the BMDCs were stained with anti-CD40-PE, antiCD80-PE, anti-CD86PE and anti-MHCII-PE (eBioscience, California, USA) and then measured by using a FACSCanto flow cytometer (BD, New York, USA).

\section{DEX isolation and identification}

For in vitro and in vivo experiments, we isolated exosomes from approximately $1 \times 10^{6}$ BMDCs cultured in media containing the different supernatants described above for $24 \mathrm{~h}$. We performed differential centrifugation to isolate exosomes from the conditioned medium [25]. Initial spins consisted of a 10-min spin at $1,000 \mathrm{~g}$, followed by a 2, $000 \mathrm{~g}$ spin for $10 \mathrm{~min}$ and a $10,000 \mathrm{~g}$ spin for $30 \mathrm{~min}$. The supernatant was retained each time. The supernatant was then centrifuged at 100, $000 \mathrm{~g}$ for $70 \mathrm{~min}$, and the pellet was resuspended in $1 \mathrm{ml}$ of PBS to dilute the remaining soluble factors, followed by another centrifugation at $100,000 \mathrm{~g}$ for $70 \mathrm{~min}$. The final pellet contained the exosomes, which were resuspended in culture media. All experiments were performed under sterile conditions. The exosomes were subsequently evaluated by transmission electron microscopy (TEM), WB and nanoparticle tracking analysis (NTA).

\section{Electron microscopy}

After a standard staining procedure with $0.5 \%$ uranyl acetate [25], TEM was performed on a Joel 1010 transmission electron microscope (JEOL Ltd, UK).

Nanoparticle tracking analysis (NTA)

Based on the rate of Brownian movement of nanoparticles in solution according to their sizes, NTA was applied [26]. Briefly, we directed a 405-nm laser light at a fixed angle to the vesicle suspension and captured 


\section{Cellular Physiology Cell Physiol Biochem 2018;46:740-756 \\ \begin{tabular}{l|l} 
and Biochemistry Published onlIne: April 05, 2018 & $\begin{array}{l}\text { D) } 2018 \text { The Author(s). Published by S. Karger AG, Basel } \\
\text { www.karger.com/cpb }\end{array}$ \\
\hline
\end{tabular}}

Zheng et al.: Dexs Attenuate Liver Ischemia Reperfusion Injury by Modulating the Balance of Treg and Th17

the scattered light with a microscope and high-sensitivity camera. By tracking the movement of individual nanoparticles over time in exosome preparations, we calculated exosome diameters using Nanosight LM10HS software (Malvern Instruments Ltd, Worcestershire, England). We captured and analyzed five recordings of $30 \mathrm{~s}$ each and analyzed data from at least 5000 individual particle tracks per sample.

\section{Isolation and quantification of naïve T cells}

We used magnetic-activated cell sorting (MACS, Miltenyi Biotec, Germany) to isolate naïve T cells. Following the manufacturer's protocol, the negative selection of naïve T cells from eight-week-old C57BL mice spleens $(n=6)$ was performed. Then, the cells were stained with anti-CD4-FITC (BioLegend, San Diego, USA) and examined with the FACS Canto flow cytometer (BD, New York, USA).

\section{Th17 and Treg cell differentiation}

We cultured purified naïve CD4+ T cells $\left(2 \times 10^{5}\right)$ in a plate pre-coated with anti-CD3 $(5 \mu \mathrm{g} / \mathrm{mL})$ and antiCD28 antibodies ( $2 \mu \mathrm{g} / \mathrm{mL}$ ) (BioLegend, San Diego, USA). For Th17 cell differentiation, we incubated naïve CD4+ T cells with TGF-B1 (5 ng/mL), IL-1 (20 ng/mL), IL-6 (20 ng/mL) and IL-23 (25 ng/mL) (PeproTech, New Jersey, USA) in the presence or absence of DEXs. After 1, 6, 12, and $24 \mathrm{~h}$ of differentiation, we stained the Th17 cells with anti-CD4-APC, anti-CD3-FITC and anti-IL-17-PE (BioLegend, San Diego, USA) and analyzed these cells by flow cytometer. Additionally, for the differentiation of Tregs, a plate previously coated with anti-CD3 $(5 \mu \mathrm{g} / \mathrm{mL})$ and anti-CD28 antibodies $(2 \mu \mathrm{g} / \mathrm{mL})$ (BioLegend, San Diego, USA) was used to culture purified CD4+ lymphocytes with TGF- $\beta 1$ ( $5 \mathrm{ng} / \mathrm{mL})$ and IL-2 (500 IU/mL) (PeproTech, New Jersey, USA) in the presence or absence of DEXs. After 1, 6, 12, and $24 \mathrm{~h}$ of differentiation, the cells were labeled with anti-CD4-FITC, anti-CD25-APC and anti-FoxP3-PE (BioLegend, San Diego, USA) and analyzed using the FACS Canto flow cytometer (BD, New York, USA).

CD4+ T cell proliferation assays

Following the manufacturer's protocol, we measured CD4+ T cell proliferation by staining purified CD4+ T cells with carboxyfluorescein diacetate succinimidyl ester (CFSE, eBioscience, California, USA). We cultured $1 \times 10^{6}$ naïve CD4+ T cells for $24 \mathrm{~h}$ and stimulated these cells with phytohemagglutinin M (PHA-M, 5 $\mu \mathrm{g} / \mathrm{mL}$, Sigma-Aldrich, Missouri, USA) in the presence or absence of DEXs. After $24 \mathrm{~h}$, we harvested the cells and analyzed the samples by flow cytometer.

\section{Interaction of CD4+ T cells and DEXS}

We labeled DEXs with PKH67 (Sigma-Aldrich, Missouri, USA) following the manufacturer's protocol and incubated $100 \mu$ lof PKH67-labeled DEXs with $5 \times 10^{4} \mathrm{CD} 4+\mathrm{T}$ cells in $0.5 \mathrm{~mL}$ of medium for $24 \mathrm{~h}$. At 1 , 6,12 , and $24 \mathrm{~h}, \mathrm{CD} 4+\mathrm{T}$ cells were fixed with $4 \%$ paraformaldehyde and stained with Dil (Sigma-Aldrich, Missouri, USA). A Leica TCS SP8 Confocal Laser Scanning Microscope (Leica Microsystems, Germany) was used to analyze the samples.

For the DEX uptake experiments, DEXs were labeled with a PKH67 Green Fluorescent Cell Linker kit (Sigma-Aldrich, Missouri, USA) as previously described [16]. Briefly, we diluted DEXs in PBS and added these cells to $1 \mathrm{ml}$ of Diluent C (Sigma-Aldrich, Missouri, USA). In parallel, we added $4 \mu \mathrm{l}$ of PKH67 to $1 \mathrm{ml}$ of diluent $\mathrm{C}$ and incubated this mixture with the DEX solution for $4 \mathrm{~min}$. Subsequently, $2 \mathrm{ml}$ of 0.5\% BSA/PBS was added to bind any excess dye. Finally, we washed the labeled DEXs, diluted these cells in $100 \mu$ of PBS and used this suspension for the uptake experiments.

CD4+ T cells were fixed with 4\% paraformaldehyde and stained with PKH26 (Life Technologies, California, USA). We used the Leica microscope as described above to analyze the cells. The Leica application suite Advanced Fluorescence Lite and LAS AF Lite software (NIH) were used to capture and analyze the images.

\section{Analysis of cytokines}

We used ELISA kits from BD Bioscience (New Jersey, USA) according to the manufacturer's protocols to examine the level of serum transaminase (ALT and AST) and the release of inflammatory cytokines (IL-10 and IL-17).

\section{Incubations with chemical inhibitors}

Exosomes were added to cells and incubated for $30 \mathrm{~min}$. Where indicated, inhibitors were added to the cells $30 \mathrm{~min}$ prior to and during exosome treatment. Inhibitors were used the concentrations according to Huijts et al. [27]. Exosomes were pretreated with antibodies (anti-cmHsp70.1) for $30 \mathrm{~min}$ at a ratio of 1 


\section{Cellular Physiology Cell Physiol Biochem 2018;46:740-756 \begin{tabular}{l|l} 
and Biochemistry POI: 10.1159/000488733 & $\begin{array}{l}\text { C) } 2018 \text { The Author(s). Published by S. Karger AG, Basel } \\
\text { www.karger.com/cpb }\end{array}$
\end{tabular}}

Zheng et al.: Dexs Attenuate Liver Ischemia Reperfusion Injury by Modulating the Balance of Treg and Th17

$\mu \mathrm{g}$ antibody per $5 \mu \mathrm{g}$ exosomes. For signaling experiments, exosomes were acutely added for different times, as indicated, and the cells were rapidly lysed on ice [28].

\section{Flow cytometry}

For measuring maturation of BMDCs, we stained the BMDCs with anti-CD11c-PerCP/Cy5, anti-CD40-PE, anti-CD80-PE, anti-CD86PE and anti-MHCIIPE (eBioscience, California, USA) and analyzed these by flow cytometer. For measuring differentiation of Tregs and Th17 cells, the flow cytometer was utilized. Tregs were analyzed by flow cytometry using FITClabeled antibodies against CD4, APC-labeled antibodies against CD25 and PE-labeled antibodies against Foxp3 (Biolegend, San Diego, USA). Th17 cells were stained with anti-CD4 APC, anti-CD3 Alexa Fluor 488-A and anti-IL17 PE (Biolegend, San Diego, USA). Staining was performed in PBS supplemented with $0.1 \%$ BSA and $0.02 \%$ sodium azide for $30 \mathrm{~min}$ at $4^{\circ} \mathrm{C}$.

Intracellular and nuclear transcription factor staining was performed according to the manufacturer's protocol (Biolegend, San Diego, USA). The cells were examined on the FACS Canto flow cytometer and analyzed by Flow Jo v10.0.7 software.

\section{RT-qPCR and western blotting $(W B)$}

Total RNA was extracted from cells and organs using Trizol reagent (Invitrogen, California, USA) according to the manufacturer's protocol. Reverse transcription was performed using HiScript Q RT SuperMix for qPCR (Vazyme, Nanjing, China) following the manufacturer's protocol. Real-time quantitative PCR analysis was performed using a SYBR RT-PCR kit (Vazyme, Nanjing, China) and the LightCycler 480II Real-Time PCR system (Roche, California, USA). The primers for the genes IFN- $\alpha$, TNF- $\alpha$, TGF- $\beta$, Foxp3, IL-1, $I L-2, I L-4, I L-5, I L-6, I L-10, I L-12, I L-17$ and $\beta$-actin are listed in Table 1 . The data were normalized to the expression levels of mouse $\beta$-actin.

Protein was isolated with RIPA lysis buffer (Beyotime, Shanghai, China). Total protein levels were quantified using a Bio-Rad (Hercules, CA, USA) protein assay, and equal amounts of protein were loaded and separated using 10\% (CD63, CD81, p-AKT, HSP-70) or 8\% (p-mTOR) SDS-PAGE (Beyotime, Shanghai, China), followed by immunoblotting with the appropriate antibodies. The following antibodies were used: anti-CD63, 1:200 (Santa Cruz, Texas, USA), anti-CD81, 1:1000 (Abcam, Massachusetts, USA), anti-PI3K, 1:1000 (Cell Signaling Technology, Danvers, MA, USA), anti-p-AKT, 1:1000 (Cell Signaling Technology, Massachusetts, USA), anti-HSP-70, 1:1000 (Abcam, Massachusetts, USA) and anti-p-mTOR, 1:1000 (Cell Signaling Technology, Massachusetts, USA). After incubation with primary antibody overnight, the membranes were washed and incubated with HRP-conjugated secondary antibodies (Cell Signaling Technology, Massachusetts, USA). Then, a chemiluminescence kit (Thermo fisher, California, USA) was used to detect the fluorescence. The WB were quantified using Image Lab (Bio Rad, California, USA).

\section{Statistical analysis}

Data are expressed as the means \pm standard deviation (S.D.). We used the Mann-Whitney U test, Student's t test and one-way ANOVA to compare treatment groups. Statistical analysis was performed
Table 1. List of oligonucleotide primer pairs used in RT-qPCR analysis. all the primer sequences are referred to mouse

\begin{tabular}{|c|c|c|c|}
\hline Target Gene & Sense Primer $\left(5^{\prime}-3^{\prime}\right)$ & Antisense Primer (5'-3') & Annealing temperature \\
\hline IFN- $\gamma$ & ATGAACGCTACACACTGCATC & CCATCCTTTTGCCAGTTCCTC & $59^{\circ} \mathrm{C}$ \\
\hline TNF- $\alpha$ & GACGTGGAACTGGCAGAAGAG & TTGGTGGTTTGTGAGTGTGAG & $59^{\circ} \mathrm{C}$ \\
\hline TGF- $\beta$ & TCTGCATTGCACTTATGCTGA & AAAGGGCGATCTAGTGATGGA & $59^{\circ} \mathrm{C}$ \\
\hline Foxp3 & CACCTATGCCACCCTTATCCG & CATGCGAGTAAACCAATGGTAGA & $59^{\circ} \mathrm{C}$ \\
\hline IL-1 $\alpha$ & GAAATGCCACCTTTTGACAGTG & TGGATGCTCTCATCAGGACAG & $59^{\circ} \mathrm{C}$ \\
\hline IL-2 & TGAGCAGGATGGAGAATTACAGG & GTCCAAGTTCATCTTCTAGGCAC & $59^{\circ} \mathrm{C}$ \\
\hline IL-4 & ATCATCGGCATTTTGAACGAGG & TGCAGCTCCATGAGAACACTA & $59^{\circ} \mathrm{C}$ \\
\hline IL-5 & CTCTGTTGACAAGCAATGAGACG & TCTTCAGTATGTCTAGCCCCTG & $59^{\circ} \mathrm{C}$ \\
\hline IL-6 & TAGTCCTTCCTACCCCAATTTCC & TTGGTCCTTAGCCACTCCTTC & $59^{\circ} \mathrm{C}$ \\
\hline IL-10 & GCTCTTACTGACTGGCATGAG & CGCAGCTCTAGGAGCATGTG & $59^{\circ} \mathrm{C}$ \\
\hline IL-12 & GTCCTCAGAAGCTAACCATCTCC & CCAGAGCCTATGACTCCATGTC & $59^{\circ} \mathrm{C}$ \\
\hline IL-17 & TTTAACTCCCTTGGCGCAAAA & CTTTCCCTCCGCATTGACAC & $59^{\circ} \mathrm{C}$ \\
\hline$\beta$-actin & GAGACCTTCAACACCCCAGC & ATGTCACGCACGATTTCCC & $59^{\circ} \mathrm{C}$ \\
\hline
\end{tabular}


Zheng et al:: Dexs Attenuate Liver Ischemia Reperfusion Injury by Modulating the Balance of Treg and Th17

using SPSS (IBM, Armonk, New York, USA) and Graph Pad Prism 6 (GraphPad Software Inc., California, USA). A $p$-value $<0.05$ was considered statistically significant.

\section{Results}

Supernatants from $H / R$ primary hepatocytes promote DC maturation

Based on flow cytometric analysis, cultured bone marrowderived dendritic cells (BMDCs) had a purity of approximately 90\% (Fig. 2A and B). Supernatants from hypoxic/reoxygenation $(\mathrm{H} / \mathrm{R})$ primary hepatocytes were applied to BMDCs to mimic the IR microenvironment. BMDCs were exposed to supernatants from $\mathrm{H} / \mathrm{R}$ primary hepatocytes $(\mathrm{H} / \mathrm{R}$ group: H/R-BMDCs) and normal liver cells (Control group: CONBMDCs) for $24 \mathrm{~h}$. Compared with the unstimulated group (Negative control group: NEG-BMDCs), the expression of the DC maturation markers CD40, CD80, CD86 and MHCII was substantially increased after exposure to the supernatants from $H / R$ primary hepatocytes (Fig. 2C).

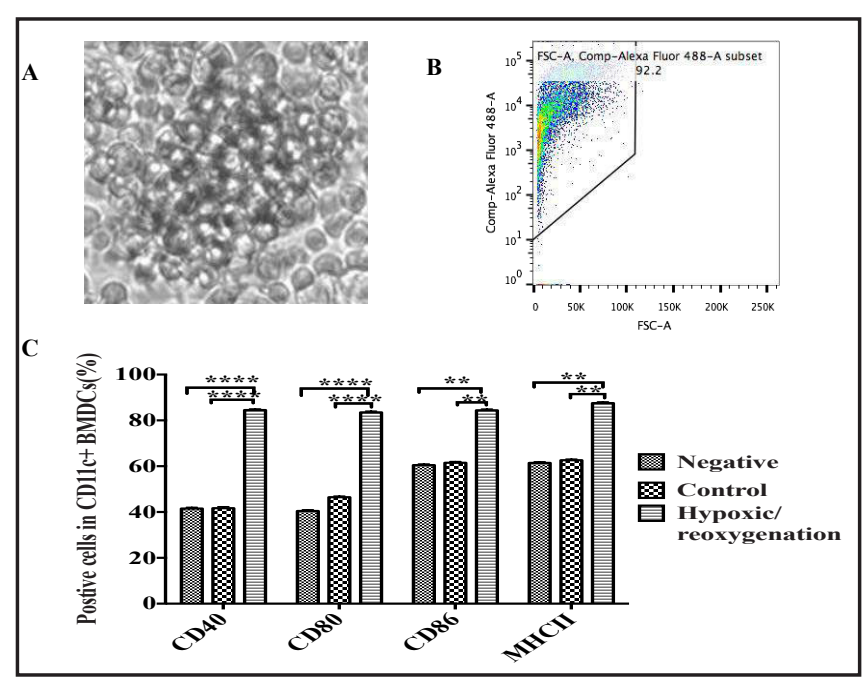

Fig. 2. Supernatants from $H / R$ primary hepatocytes upregulate BMDCs maturation. Cultured BMDCs were either unstimulated (Negative group) or stimulated with the supernatants of hypoxic/reoxygenation primary hepatocytes (H/R group), or normal hepatocytes (Control group) for $24 \mathrm{~h}$. (A) Representative image of BMDCs was captured by inverted microscope. (B) BMDCs were stained with anti-CD11c-PerCP/Cy5 and analyzed by flow cytometer. (C) Flow cytometer results showing that expression of DCs maturation markers (CD40, CD80, CD86 and MHCII) was increased after stimulation with supernatants from H/R primary hepatocytes. Data is presented as means \pm SEM, $n=6$ per group; statistical significance: ${ }^{*} \mathrm{p}<0.05 ;{ }^{* *} \mathrm{p}<0.01,{ }^{* * *} \mathrm{p}<0.001$, ${ }^{* * * *} \mathrm{p}<0.0001$. The pictures show the representative results from six independent experiments.
Fig. 3. BMDCs ameliorate liver IR injury. BMDCs from different groups were injected through the tail vein $1 \mathrm{~h}$ before liver IR. (A) $\mathrm{H} / \mathrm{R}-\mathrm{BMDC}$ reduced liver IR injury to a greater extent than NEG/ CON-BMDCs. To analyze infarct size and morphology, (B) Suzuki's scores showed that H/R-BMDCs relieved in vivo IR injury more effectively than CON-BMDCs or NEG-BMDCs, (C, D) and also reduced serum transaminase levels to a greater extent. Data is presented as means \pm SEM, $n=6$ mice per group; statistical significance: ${ }^{*} \mathrm{p}<0.05 ; \quad{ }^{* *} \mathrm{p}<0.01, \quad{ }^{* * *} \mathrm{p}<0.001$, $* * * * \mathrm{p}<0.0001$. The pictures show the representative results from six independent experiments.

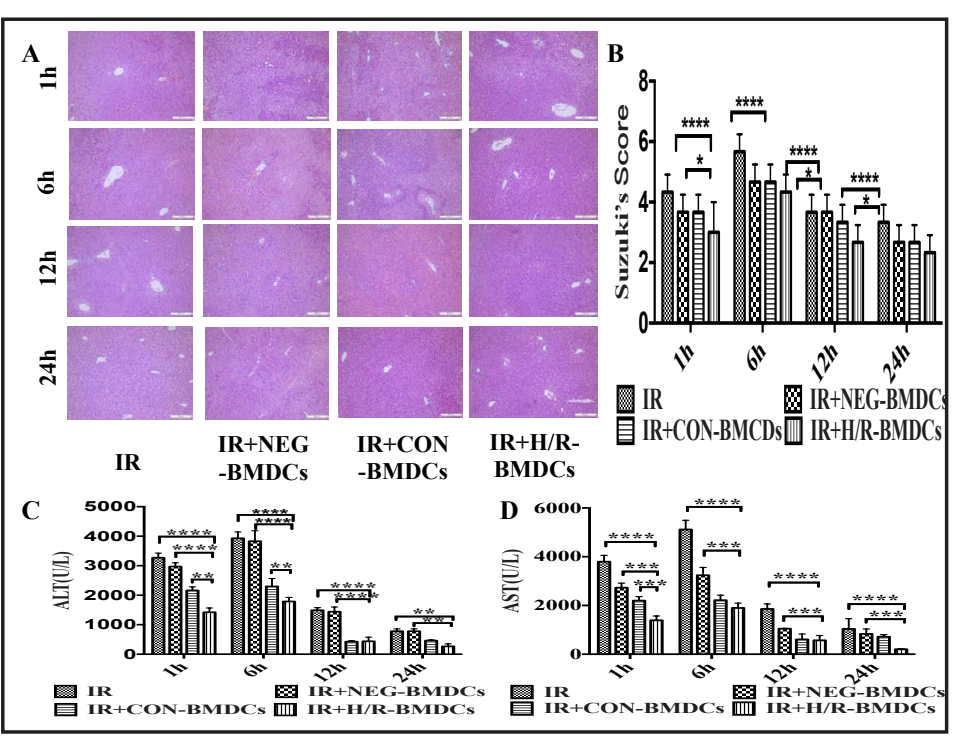


Mature DCs alleviated liver I/R injury by modulating the balance between Treg and Th17 cells

To determine the impact of DCs in liver I/R, we injected BMDCs through the mouse tail vein at $1 \mathrm{~h}$ prior to I/R. Morphometric analysis and Suzuki's scores (Fig. 3A and B) showed that H/R BMDCs improved liver IRI to a greater extent than BMDCs from the negative and control groups. Additionally, the results of serum transaminase tests (ALT and AST, Fig. 3C and D) showed a significant decrease in $H / R$.

Furthermore, male wild-type mice were treated with NEG-BMDCs, CON-BMDCs or H/R-BMDCs at $1 \mathrm{~h}$ prior to liver I/R. At $6 \mathrm{~h}$ after liver IR, RT-qPCR results showed that H/R BMDCs induced significant upregulation of the anti-inflammatory cytokines TGF- $\beta$, Foxp3 and interleukin (IL)-10 and downregulation of IL-17, compared with the CON-BMDC and NEG-BMDC groups. Other inflammatory cytokines, such as TNF- $\alpha$, IFN- $\alpha$, IL-1, IL-2, IL-4, IL5, IL-6 and IL-12, were not significantly different among the BMDC groups (Fig. 4A). These results demonstrate that BMDCs can relieve liver IR injury in part by modulating cytokine expression from Tregs and Th17 cells.

In the present study, based on the aforementioned RT-qPCR results, we analyzed serum IL-10 and IL-17 by ELISA. We observed that serum IL-10 in the H/R-BMDC group was significantly higher than that in the control and negative groups, whereas the concentration of serum IL-17 displayed the opposite pattern of expression compared to IL-10 (Fig. 4 B and C).

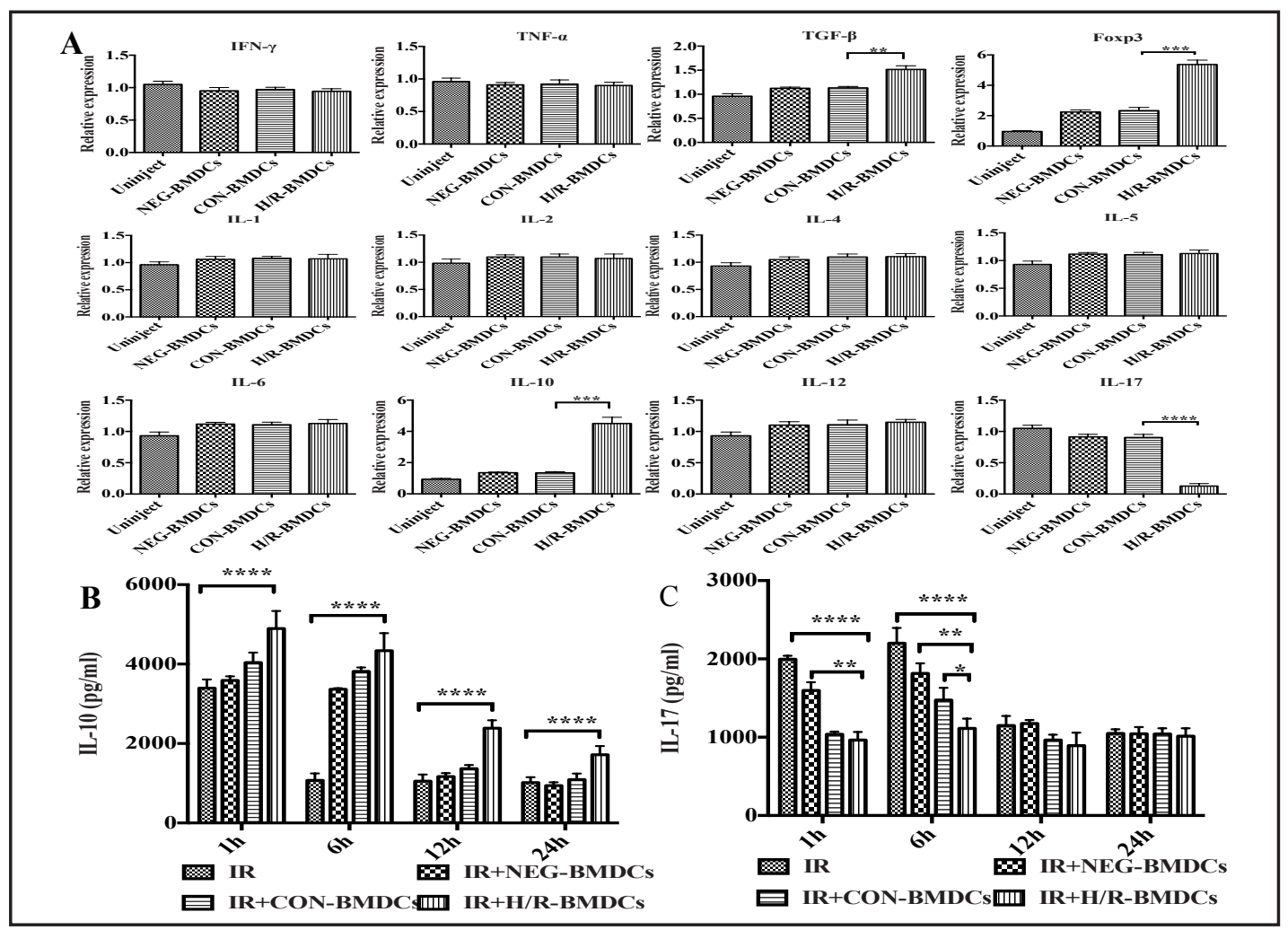

Fig. 4. BMDCs modulated the relative mRNA expression of inflammatory cytokines. The male wild-type mice were either untreated or treated with NEG-BMDCs, CON-BMDCs or H/R-BMDCs at $1 \mathrm{~h}$ before liver IR. (A) At $6 \mathrm{~h}$ after liver I/R, H/R-BMDCs induced significant upregulation in levels of anti-inflammatory cytokines TGF- $\beta$, Foxp3 and IL-10, and downregulation of IL-17 compared with the CON-BMDC and NEGBMDC groups. Other inflammatory cytokines, including TNF- $\alpha$, IFN- $\alpha$, IL-1, IL-2, IL-4, IL-5, IL-6 and IL-12 did not show any significant differences among the BMDC groups. (B, C) ELISA of serum inflammatory cytokines showed that H/R-BMDCs increased serum IL-10 and downregulated serum IL-17. Data is presented as means $\pm S E M, n=6$ mice per group; statistical significance: ${ }^{*} p<0.05 ;{ }^{* *} p<0.01,{ }^{* * *} p<0.001,{ }^{* * * *} p<0.0001$. The pictures show the representative results from six independent experiments. 
Fig. 5. BMDCs modulated the balance between Tregs and Th17 cells via DEXs. We performed the co-culture of BMDCs and naïve T cells and differentiation of Tregs and Th17 cells. H/R-BMDCs upregulated the differentiation of Tregs and inhibited the differentiation of Th17 cells. Then, UW4869 was used to inhibit DEXs release. According to ELISA and flow cytometry, the ratio of Tregs to Th17 cells in the UW4869-treated group was significantly lower than in the con-

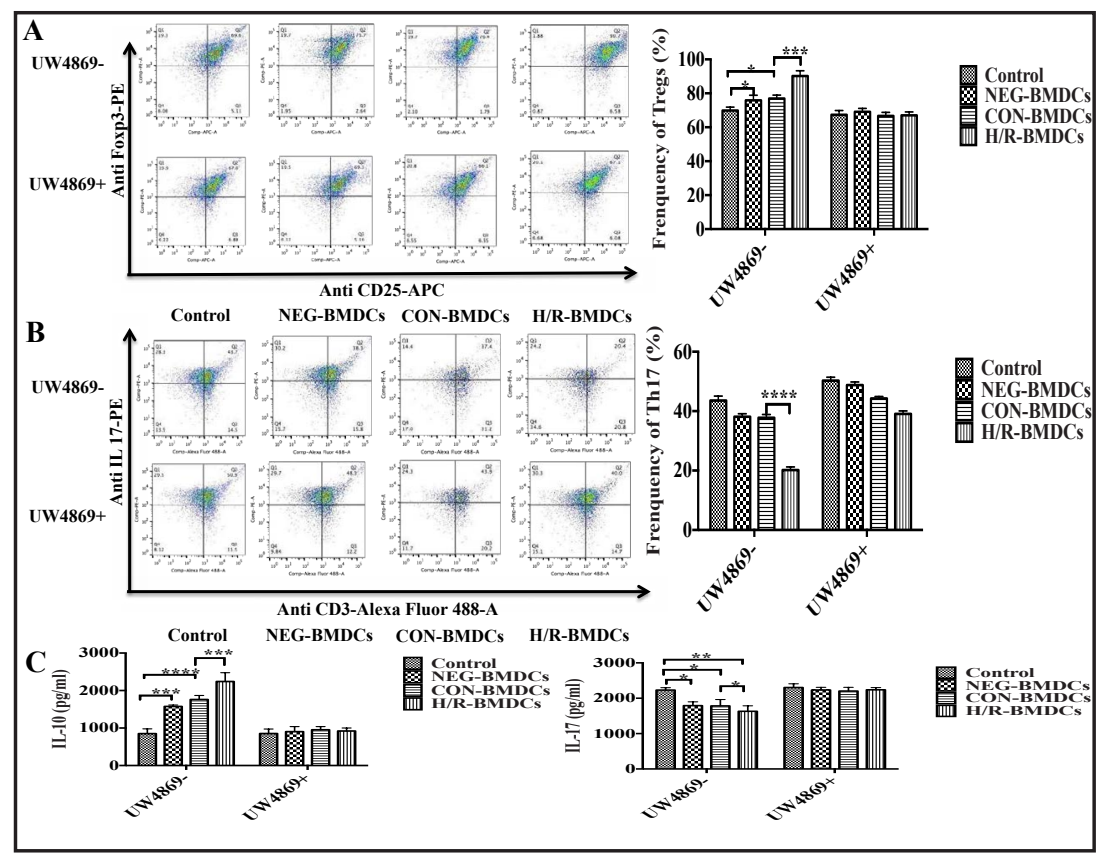
trol group, whereas the ratio of Th17 cells to Tregs was considerably higher. Data is presented as means \pm SEM, $n=6$ per group; statistical significance: ${ }^{*} \mathrm{p}<0.05 ;{ }^{* *} \mathrm{p}<0.01,{ }^{* * *} \mathrm{p}<0.001,{ }^{* * *} \mathrm{p}<0.0001$. The pictures show the representative results from six independent experiments.

Fig. 6. DEXs were isolated by ultracentrifugation and characterized by transmission electron microscopy (TEM), western blotting and NTA. DEXs characterization by TEM (A) (scale bar, $100 \mathrm{~nm}$ ) and western blotting (B) ( Medium alone is the control). (C, D) NTA demonstrated the size distribution and concentration of DEXs from the different groups. There were more and larger particles in the medium from the H/R-BMDC group than in media from the CON-BMDC and NEG-BMDC groups. Data is presented as means \pm SEM, $\mathrm{n}=6$ per group; statistical significance: ${ }^{*} \mathrm{p}<0.05 ; \quad * * \mathrm{p}<0.01, \quad * * * \mathrm{p}<0.001$,

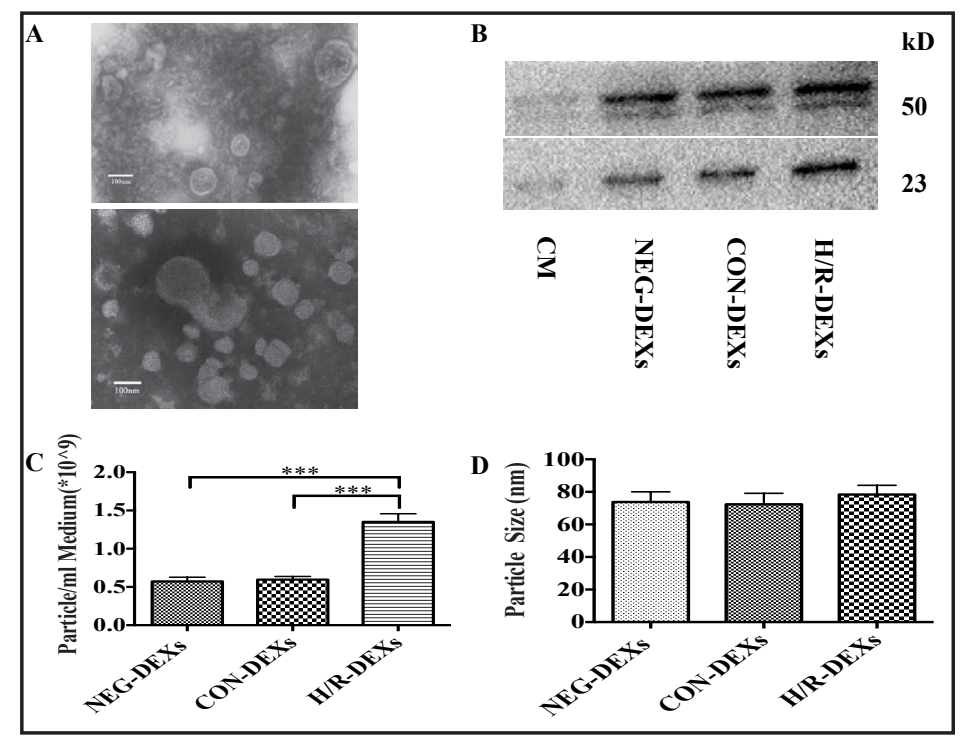
$* * * * \mathrm{p}<0.0001$. The pictures show the representative results from six independent experiments.

BMDCs modulated the balance between Tregs and Th17 cells via DEXs

The cytokine analysis results suggested that BMDCs could alter the ratio of Tregs to Th17 cells. To further verify that BMDCs could regulate these immune cells, the co-culture of BMDCs and naïve T cells was performed using 0.4- $\mu \mathrm{m}$ Transwell culture plates. According to flow cytometer, H/R-BMDCs upregulated the differentiation of Tregs and inhibited the differentiation of Th17 cells (Fig. 5A and B). 


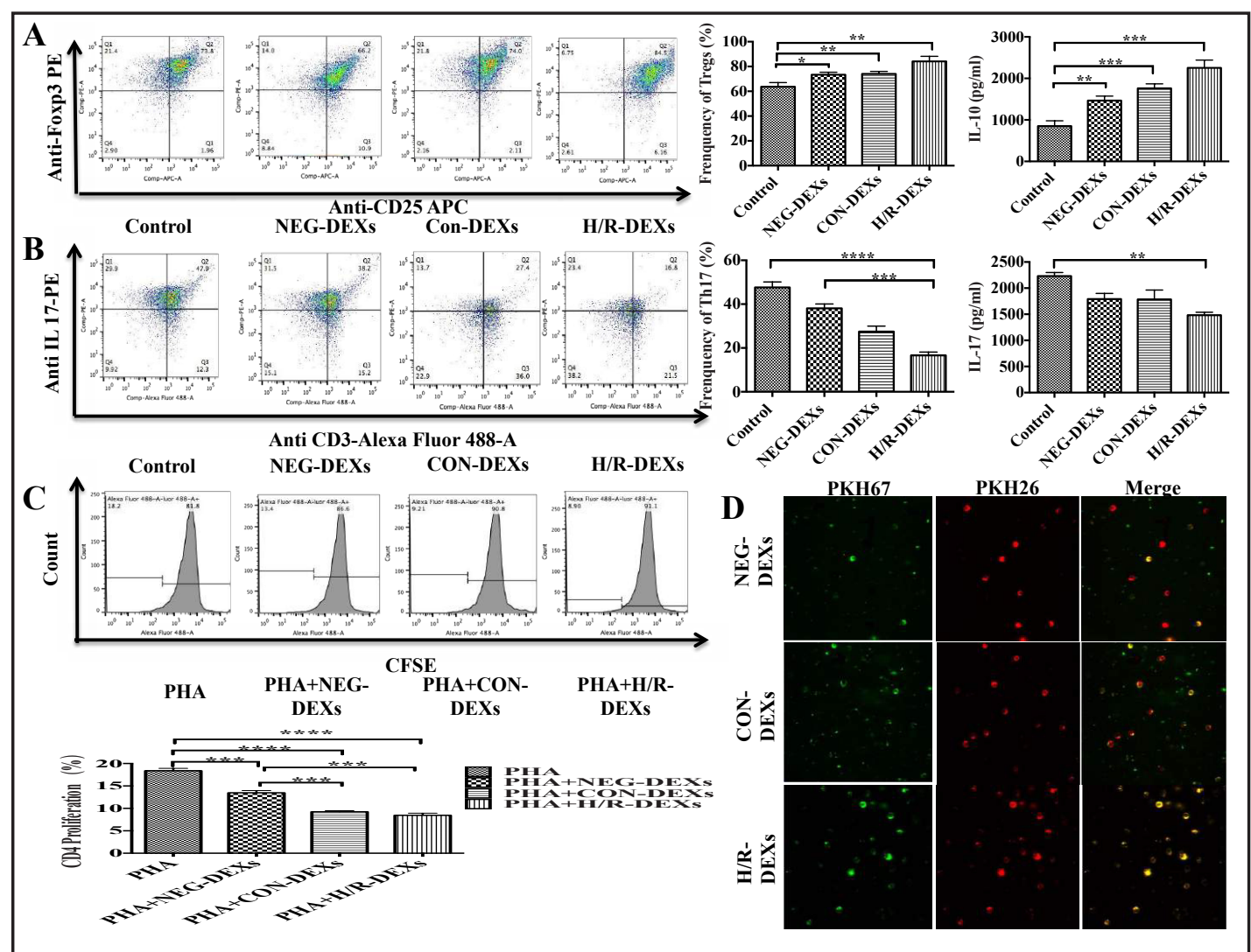

Fig. 7. DEXs modulated the differentiation of Tregs and Th17 cells. We prepared co-cultures of naïve $\mathrm{T}$ cells and DEXs to induce the differentiation of Tregs and Th17 cells. (A) DEXs derived from the H/R group promoted the differentiation of Tregs and the production of IL-10 more effectively than did DEXs from the control and negative groups. (B) DEXs derived from the H/R group inhibited the differentiation of Th17 cells and production of IL-17 more effectively than it in control and negative groups. (C) Naïve T cells were stained with CSFE and cultured for $24 \mathrm{~h}$ with PHA-M and/or different DEXs. H/R-DEXs significantly repressed CD4+ T cell proliferation $24 \mathrm{~h}$ following phytohemagglutinin stimulation, whereas no significant differences in proliferation were found after CON-DEX or NEG-DEX stimulation or after no stimulation. (D) Uptake of DEXs by naïve T cells. DEXs were labeled with PKH67 (green), and naïve T cells were stained with the membrane dye PKH26 (red). Confocal imaging was used to observe the cells. Uptake of H/R-DEXs by T cells was clearly increased compared to uptake of CON-DEXs or NEG-DEXs. Data is presented as means \pm SEM, $\mathrm{n}=6$ per group; statistical significance: ${ }^{*} \mathrm{p}<0.05 ;{ }^{* *} \mathrm{p}<0.01,{ }^{* * *} \mathrm{p}<0.001,{ }^{* * *} \mathrm{p}<0.0001$. The pictures show the representative results from six independent experiments.

To determine the function of DEXs, UW4869 was used to inhibit the release of DEXs. Based on ELISA and flow cytometry, the ratio of Tregs to Th17 cells in the UW4869-treated group was significantly lower than that in the control group, whereas the ratio of Th17 cells to Tregs was considerably higher (Fig. 5A, B and C). These results demonstrate that DCs can modulate the balance between Tregs and Th17 cells, which was dependent on the release of DEXs.

\section{DEXs modulated the differentiation of Tregs and Th17 cells}

To investigate the impact of DEXs on naïve T cells, we isolated DEXs as previously described [26] and characterized these cells by transmission electron microscopy and WB (Fig. 6A and B). NTA was used to examine the concentration and size of DEXs. The results showed that there were more and larger particles in the medium from the H/R-BMDC group than in the media from the CON-BMDC and NEG-BMDC groups (Fig. 6C and D). We

\section{KARGER}




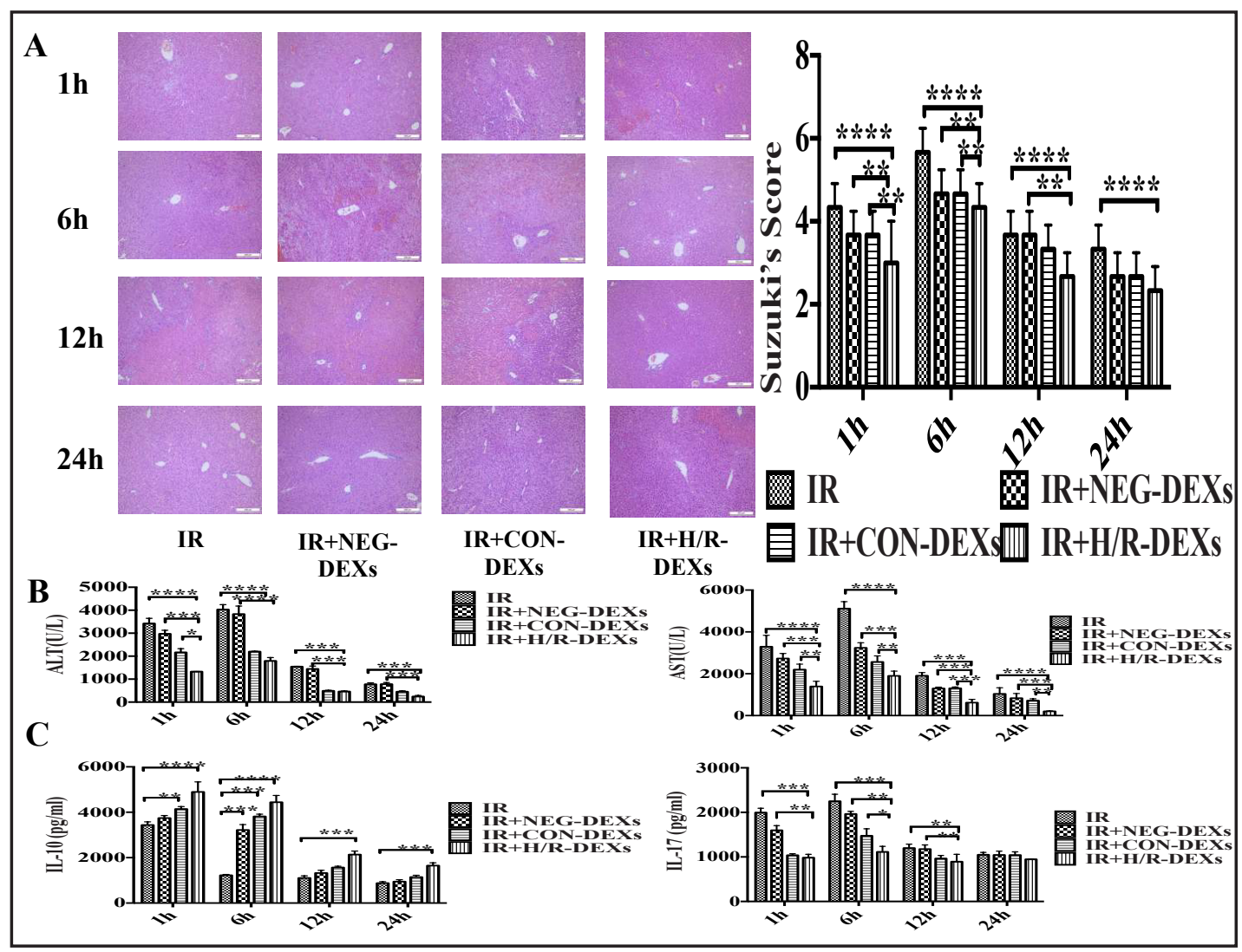

Fig. 8. Injected DEXs improved liver function in mice post-IR. DEXs were injected through the tail vein $1 \mathrm{~h}$ before liver IR. (A-B) To analyze infarct size and morphology, Suzuki's scores showed that H/R-DEXs relieved IR injury more completely than CON-DEXs or NEG-DEXs, and produced a greater decrease in serum aminopherase levels. (C) ELISA of serum inflammatory cytokines showed that H/R-DEXs increased serum IL-10 and downregulated serum IL-17. Data is presented as means \pm SEM, $n=6$ mice per group; statistical significance: ${ }^{*} \mathrm{p}<0.05 ;{ }^{* *} \mathrm{p}<0.01,{ }^{* * *} \mathrm{p}<0.001,{ }^{* * *} \mathrm{p}<0.0001$. The pictures show the representative results from six independent experiments.

then prepared co-cultures of naïve T cells and DEXs to induce the differentiation of Tregs and Th17 cells. We found that the number of Tregs in the H/R-DEX group was significantly greater than that in the CON-DEX and NEG-DEX groups (Fig. 7A), while the number of Th17 cells was lower in the H/R-DEX group compared to that in the other groups (Fig. 7B).

We also investigated whether DEXs could modulate CD4+ T cell proliferation. We observed that H/R-DEXs significantly repressed CD4+ T cell proliferation at $24 \mathrm{~h}$ following phytohemagglutinin stimulation, whereas no significant differences in proliferation were found after CON-DEX or NEG-DEX stimulation or after no stimulation (Fig. 7C).

Finally, we used confocal imaging to observe the uptake of DEXs. A separate comparison of the negative, control and H/R groups was conducted. We found that uptake of H/R-DEXs by naïve T cells was significantly greater than the uptake of CON-DEXs or NEG-DEXs (Fig. 7D).

Injected DEXs improved liver function in mice post-IR

To verify the function of DEXs in liver IR injury, we injected DEXs into mice through the tail vein and examined the histological differences between the different groups at 1, 6, 12 and $24 \mathrm{~h}$ post-IR. According to morphometric analysis and Suzuki's scores, we found that IR injury was reduced in the H/R-DEX group compared to the CON-DEX, NEG-DEX and noninjected groups (Fig. 8A). In addition, H/R-DEXs downregulated the levels of serum ALT and 


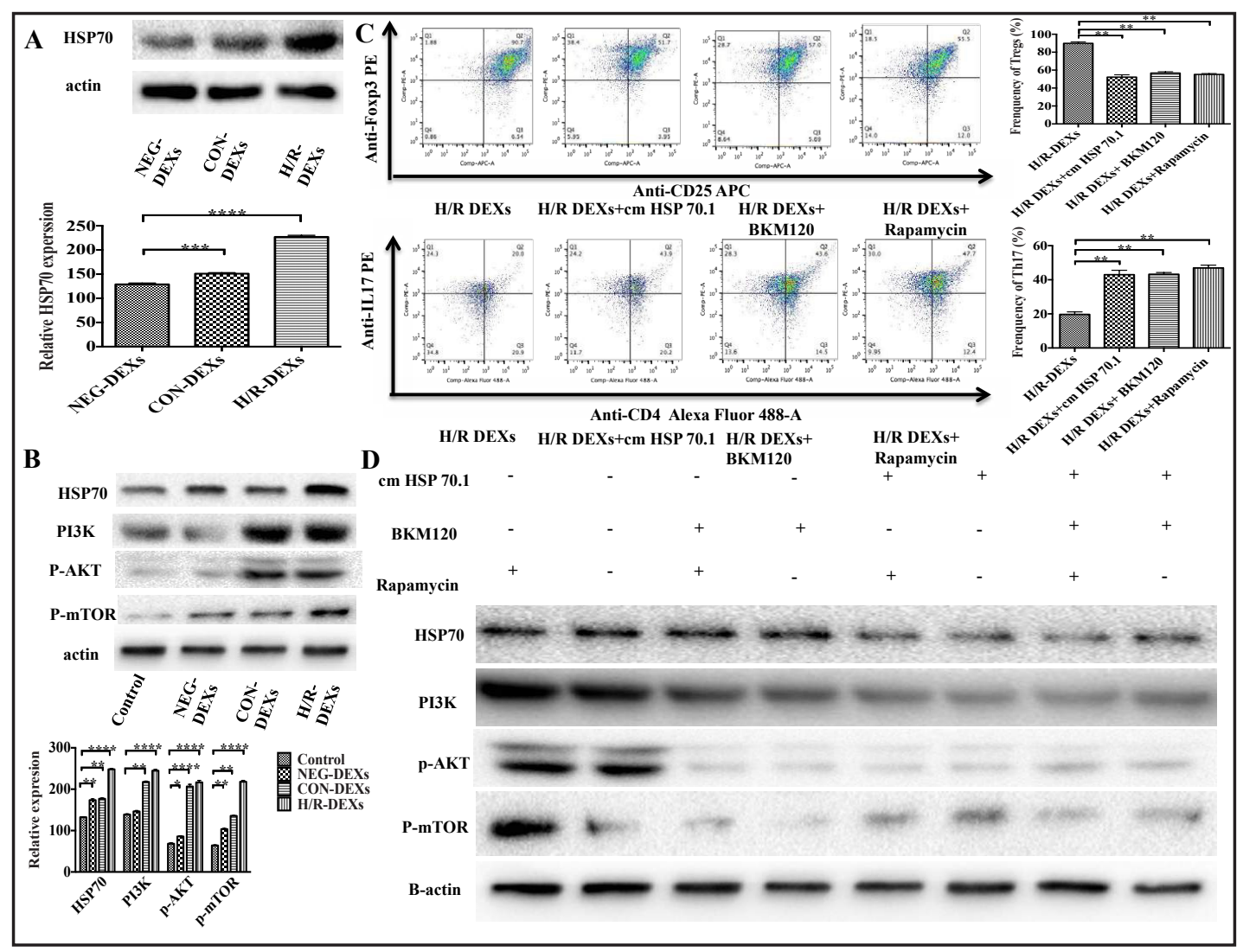

Fig. 9. DEXs mediated the transport of HSP 70 to naïve T cells and modulated the differentiation of Tregs and Th17 cells. (A) Western blotting was performed to analyze the expression of HSP70 in DEXs, which was significantly higher in H/R-DEXs compared to CON-DEXs or NEG-DEXs. (B) Promotion of PI3K expression and phosphorylation of AKT and mTOR in naïve T cells by H/R-DEXs. (C) Neutralization of HSP70 in H/RDEXs by cmHSP70.1 modulated the differentiation of Tregs and Th17 cells. In this case, an inhibiter of PI3K and mTOR limited the effect of H/R-DEXs in differentiating Tregs and Th17 cells. (D) Inhibitors of HSP70 (cm HSP 70.1), PI3K (BKM120), or mTOR (Rapamycin) block the expression of PI3K and the phosphorylation of both AKT and mTOR. On their own, BKM120 and Rapamycin decreased mTOR phosphorylation. Data is presented as means $\pm \mathrm{SEM}, \mathrm{n}=6$ per group; statistical significance: ${ }^{*} \mathrm{p}<0.05 ;{ }^{* *} \mathrm{p}<0.01,{ }^{* * *} \mathrm{p}<0.001$, $* * * * \mathrm{p}<0.0001$. The pictures show the representative results from six independent experiments.

AST, as well as inflammatory IL-17 compared with the CON-DEX, NEG-DEX and non-injection groups. Anti-inflammatory cytokines, such as IL-10, were significantly upregulated by H/RDEXs (Fig. 8B and C).

These in vivo results demonstrated that H/R-DEXs improved in vivo IR injury by modulating the balance between Treg and Th17 cell populations.

\section{DEXs mediated the transport of HSP 70 to naïve T cells}

A previous study showed that HSP70 plays a critical role in liver IR injury [15]. During kidney ischemia, CD11c+ dendritic cells and/or F4/80+ macrophages are the main source of HSP70 expression [29]. We used supernatants from H/R and normal primary hepatocytes to stimulate BMDCs. WB results showed that HSP70 expression was significantly higher in the H/R-DEX group than in the control and negative groups (Fig. 9A). Thus, we inferred that DEXs could transport HSP70 into T cells.

Furthermore, previous studies have demonstrated that HSP70 interacts with T cells mainly via toll-like receptor (TLR) 2 and TLR4. In addition, a recent study demonstrated that phosphatidylinositol 3-kinase (PI3K)/AKT, p38 and c-Jun N-terminal kinase (JNK)

\section{KARGER}




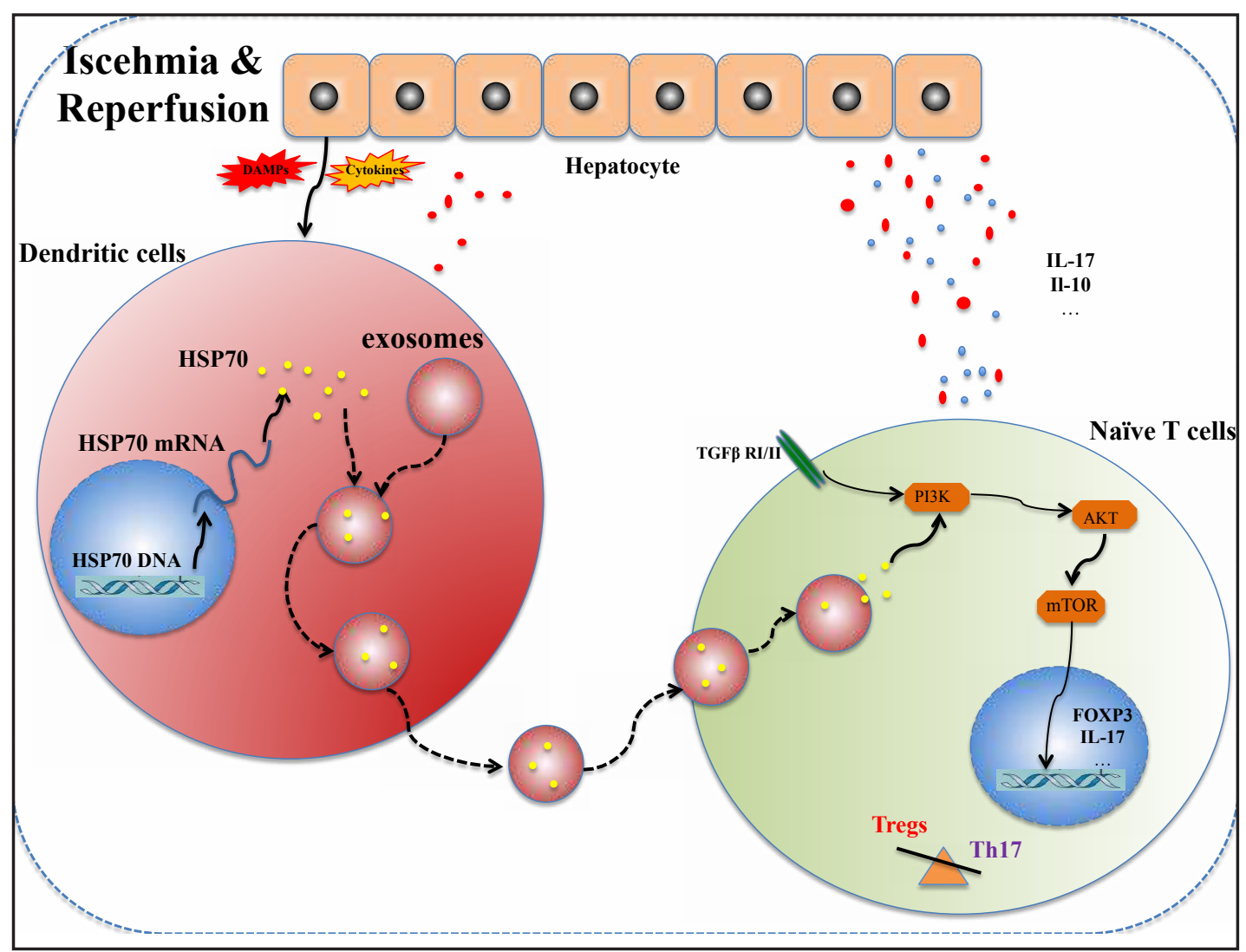

Fig. 10. Schematic of the proposed mechanism of HSP 70 in BMDCs and naïve.

mitogen-activated protein kinase (MAPK) were involved in HSP70-dependent activation of Tregs [30]. PI3K inhibition alone might offer antitumor efficacy without the detrimental selective expansion of Tregs associated with mTOR inhibition [27]. To verify the transport and function of HSP70, the co-culture of DEXs and naïve T cells was performed. WB revealed that HSP 70 promoted PI3K expression and the phosphorylation of AKT and mTOR in H/RDEX-treated naïve T cells (Fig. 9B). Then, we used inhibitors of HSP70 (anti-cmHSP70.1), PI3K (BKM120) and mTOR (Rapamycin) to inhibit the expression of HSP70, PI3K and mTOR in DEXs and naïve T cells, prepared co-cultures of DEXs and naïve T cells, and induced the differentiation of Tregs and Th17. Based on flow cytometry, cmHSP70.1, BKM120 and Rapamycin inhibited the differentiation of Tregs and promoted the differentiation of Th17 cells (Fig. 9C). In addition, there was no significant difference between the BKM120- and rapamycin-treated groups (Fig. 9C). WB showed that inhibitors of HSP70, PI3K and mTOR blocked the expression of PI3K and the phosphorylation of both AKT and mTOR. Treatment with either BKM120 or Rapamycin alone decreased mTOR phosphorylation (Fig. 9D). Thus, these results demonstrate that HSP70 modulated the differentiation of Tregs and Th17 cells via the PI3K/mTOR axis.

\section{Discussion}

In the present study, we demonstrate a novel and significant relationship between DCs and CD4+ T cells, and the function of DEXs in liver IRI. The results show that a large quantity of exosomes is secreted from BMDCs when expose to an H/R hepatocyte microenvironment, and the release of exosomes modulates the balance between Treg and Th17 cells. Furthermore, H/R-DEXs increase the differentiation of Tregs and suppress the differentiation of Th17 cells. Finally, H/R-DEXs injected into the tail vein improve mouse liver function post-liver 
IR. These results show that DEXs released during liver IRI may control the balance between Treg and Th17 cells and improve liver function, as observed by HE staining (E).

Many studies have demonstrated that the mechanism triggered by transplanted cells significantly contributes to liver IRI [31, 32]. Various proteins secreted by DCs play important roles in the improvement of liver function. In addition, a previous study demonstrated that the blockade of TIM-4 on DC significantly inhibited T helper- 2 cell differentiation, induced CD4+ CD25+ Foxp3+ T regulatory cell (iTreg) expansion and improved liver IRI [8]. In the present study, H/R primary hepatocytes are used to mimic the liver ischemic condition. The results show that supernatants from $\mathrm{H} / \mathrm{R}$ primary hepatocytes up-regulate the expression of BMDC maturation markers (CD40, CD80, CD86 and MHC-II) and also HSP70, consistent with previous studies showing that DC maturation markers increased after liver IRI [6]. Moreover, observations of serum transaminase levels and morphological changes revealed that BMDCs can ameliorate liver IR injury and that H/R-BMDCs modulated liver IR injury to a greater extent than NEG/CON-BMDCs, according to the RT-PCR results. Thus, we determined that BMDCs relieve liver IRI by modulating the numbers of Tregs and Th17 cells. The present study demonstrates the relationships among BMDCs, the balance between Tregs and Th17 cells and liver IRI.

Exosomes, as cell-free products, exhibit potential for circumventing many of the limitations of using DCs as therapeutic agents. The use of non-cell therapy methods has shown several promising prospects in reducing long-term side effects, such as arrhythmia, calcification, and multi-directional differentiation [33]. Reportedly, exosomes derived from antigen-presenting cells activate $\mathrm{T}$ and $\mathrm{B}$ cells and have been explored for their immunestimulatory properties in cancer therapy. The immunosuppressive properties of exosomes derived from macrophages and DCs reduced inflammation in animal models of several inflammatory disorders [15, 34]. In autochthonous hepatocellular carcinoma mouse models, DEXs treated in the tumor microenvironment reduced the number of Tregs and the serum concentrations of IL-10 and TGF- $\beta$ [35]. Using the exosome inhibitor (UW4869), the regulating action of BMDCs on the differentiation of Tregs and Th17 cells is inhibited, suggesting that DEXs can mediate the differentiation of Treg and Th17 cells and accelerating research to determine whether DEXs in the post-I/R hepatocyte microenvironment activate naïve T cells and modulate the balance between Tregs and Th17 cells. In the present study, DEXs accelerated differentiation of Tregs and inhibited the differentiation of Th17 cells. Furthermore, H/R-DEXs modulate the balance between Treg and Th17 cells to a greater extent than did CON-DEXs or NEG-DEXs. Additionally, confocal imaging revealed that naïve $T$ cells directly endocytosed DEXs, and there was a greater uptake of H/R-DEXs by naïve $T$ cells. Furthermore, when we injected DEXs into mice through the tail vein prior to IR injury, we observed the alleviation of IR injury by the upregulation of IL-10 and downregulation of IL-17. These in vitro and in vivo results showed that H/R-DEXs promote the differentiation of Tregs, repress the differentiation of Th17 cells and the proliferation of CD4+ T cells, and trigger homeostatic liver protection after IRI.

Previous studies have reported that HSP70 also plays a crucial role in liver IR injury [30]. In other tissue models, such as kidney IR injury, HSP70 induced reno-protective effects, which were partially mediated by CD4+CD25+Foxp3+ Treg cells [29]. The present study demonstrated that DEXs promote the differentiation of Tregs. WB results showed that the expression of HSP70 is enhanced in DEXs after stimulation in the post-IR hepatocyte microenvironment as mimicked by incubation with $H / R$ hepatocyte supernatants. Furthermore, the HSP70 inhibitor can inhibit the differentiation of Tregs. These results demonstrate that HSP70 in DEXs mediated the differentiation of Tregs.

Finally, a previous study showed that HSPs interact with T cells mainly via TLR2 and TLR4 [36]. PI3K/AKT, p38 and JNK MAPKs were also involved in the HSP70-dependent activation of Tregs, while the MAPK ERK1/2 had a much lower impact on Tregs [30]. PI3K inhibition alone might exert antitumor effects without the detrimental selective expansion of Tregs associated with mTOR inhibition [27]. Similar to these findings, we observed the activation of PI3K/AKT and mTOR after stimulation with H/R-DEXs. Furthermore, after treatment with 
the mTOR inhibitor rapamycin or the single pan PI3K inhibitor BKM120, Treg differentiation is inhibited. Thus, these results suggest that HSP70 modulated the differentiation of Tregs by stimulating the PI3K-mTOR signaling axis.

In conclusion, DEXs protect the liver from IRI by modulating the balance between Tregs and Th17 cells. Furthermore, liver IR increased the expression of HSP70 in DEXs. DEXs transported HSP70 into naïve T cells and stimulated the PI3K/mTOR axis to promote Treg differentiation (Fig. 10). Therefore, DEXs are worthy of further investigation as a potent strategy to protect the liver from IRI.

\section{Study limitations}

There were several limitations in the present study. First, while there are a number of methods for DEX isolation, it is impossible to achieve complete purity of exosomes in cellular supernatants; consequently, a significant amount of proteins and protein aggregates may still be present in the isolated fractions. Additionally, we could not exclude the potential involvement of non-exosomal HSP70 or rule out other unknown inducers of the PI3K/ mTOR axis. Second, DEXs taken up by naïve T cells modulated the differentiation of Treg and Th17 cells, and HSP70 played a critical role in this process. However, exosomes are clearly complex vesicles that contain an assortment of different membranes, soluble proteins and different types of RNAs. It is therefore difficult to address all of the mechanisms underlying the differentiation of naïve T cells by DEXs. Third, the percentage of H/R-DEXs endocytosed by naïve T cells was significantly higher than the percentage of CON-DEXs and NEG-DEXs. Additional studies are needed to confirm the role of DEX recruitment. Finally, the present study showed that DEXs improved liver IR injury by modulating the balance between Tregs and Th17 cells. However, CD4+ T cells not only include Th1 and Th2 cells but also Th17 cells, Tregs and Tfh cells, with both pro- and anti-inflammatory functions. Further studies are needed to address the function of DEXs in these other T cell subsets.

\section{Acknowledgements}

L.Z. researched data and contributed to discussion. D.Z. and Z.F. assisted animal feeding and experiments, and provided other technical assistance. L.Z. and D.Z. wrote the manuscript. L.K. and L.P. contributed to discussion and reviewed/edited manuscript. L.Z. and W.L. designed the study, and revised/edited manuscript.

This study was supported by grants from the Natural Science Foundation of China (81270483).

\section{Disclosure Statement}

No conflict of interests exists.

\section{References}

1 Honda M, Takeichi T, Hashimoto S, Yoshii D, Isono K, Hayashida S, Ohya Y, Yamamoto H, Sugawara Y, Inomata Y: Intravital Imaging of Neutrophil Recruitment Reveals the Efficacy of FPR1 Blockade in Hepatic Ischemia-Reperfusion Injury. J Immunol 2017;198:1718-1728.

-2 Ohana G, Cohen S, Rath-Wolfson L, Fishman P: A3 adenosine receptor agonist, CF102, protects against hepatic ischemia/reperfusion injury following partial hepatectomy. Mol Med Rep 2016;14:4335-4341.

-3 Tsaroucha AK, Tsiaousidou A, Ouzounidis N, Tsalkidou E, Lambropoulou M, Giakoustidis D, Chatzaki E, Simopoulos C: Intraperitoneal administration of apigenin in liver ischemia/reperfusion injury protective effects. Saudi J Gastroenterol 2016;22:415-422. 


\section{Cellular Physiology Cell Physiol Biochem 2018;46:740-756 \begin{tabular}{c|c|c|c|} 
DOI: 10.1159/000488733 & C 2018 The Author(s). Published by S. Karger AG, Basel \\
wwww.kargercom/cpb
\end{tabular}

Zheng et al.: Dexs Attenuate Liver Ischemia Reperfusion Injury by Modulating the Balance of Treg and Th17

-4 Zhang B, Liu QH, Zhou CJ, Hu MZ, Qian HX: Protective effect of eNOS overexpression against ischemia/ reperfusion injury in small-for-size liver transplantation. Exp Ther Med 2016;12:3181-3188.

5 Palumbo T, Nakamura K, Lassman C, Kidani Y, Bensinger SJ, Busuttil R, Kupiec-Weglinski J, Zarrinpar A: Bruton Tyrosine Kinase Inhibition Attenuates Liver Damage in a Mouse Warm Ischemia and Reperfusion Model. Transplantation 2017;101:322-331.

-6 Zhang M, Ueki S, Kimura S, Yoshida O, Castellaneta A, Ozaki KS, Demetris AJ, Ross M, Vodovotz Y, Thomson AW, D BS, Geller DA, Murase N: Roles of dendritic cells in murine hepatic warm and liver transplantationinduced cold ischemia/reperfusion injury. Hepatology 2013;57:1585-1596.

7 Lu L, Zhou H, Ni M, Wang X, Busuttil R, Kupiec-Weglinski J, Zhai Y: Innate Immune Regulations and Liver Ischemia-Reperfusion Injury. Transplantation 2016;100:2601-2610.

-8 Li J, Zhao X, Liu X, Liu H: Disruption of TIM-4 in dendritic cell ameliorates hepatic warm IR injury through the induction of regulatory T cells. Mol Immunol 2015;66:117-125.

-9 Kuboki S, Sakai N, Tschop J, Edwards MJ, Lentsch AB, Caldwell CC: Distinct contributions of CD4+ T cell subsets in hepatic ischemia/reperfusion injury. Am J Physiol Gastrointest Liver Physiol 2009;296:G10541059.

10 Park SW, Kim M, Brown KM, D'Agati VD, Lee HT: Paneth cell-derived interleukin-17A causes multiorgan dysfunction after hepatic ischemia and reperfusion injury. Hepatology 2011;53:1662-1675.

-11 Feng M, Wang Q, Zhang F, Lu L: Ex vivo induced regulatory T cells regulate inflammatory response of Kupffer cells by TGF-beta and attenuate liver ischemia reperfusion injury. Int Immunopharmacol 2012;12:189-196.

12 Rao J, Lu L, Zhai Y: T cells in organ ischemia reperfusion injury. Curr Opin Organ Transplant 2014;19:115120.

13 Tran TH, Mattheolabakis G, Aldawsari H, Amiji M: Exosomes as nanocarriers for immunotherapy of cancer and inflammatory diseases. Clin Immunol 2015;160:46-58.

14 Greening DW, Gopal SK, Xu R, Simpson RJ, Chen W: Exosomes and their roles in immune regulation and cancer. Semin Cell Dev Biol 2015;40:72-81.

15 Shenoda BB, Ajit SK: Modulation of Immune Responses by Exosomes Derived from Antigen-Presenting Cells. Clin Med Insights Pathol 2016;9:1-8.

16 Liu H, Gao W, Yuan J, Wu C, Yao K, Zhang L, Ma L, Zhu J, Zou Y, Ge J: Exosomes derived from dendritic cells improve cardiac function via activation of CD4(+) T lymphocytes after myocardial infarction. J Mol Cell Cardiol 2016;91:123-133.

17 Pitt JM, Andre F, Amigorena S, Soria JC, Eggermont A, Kroemer G, Zitvogel L: Dendritic cell-derived exosomes for cancer therapy. J Clin Invest 2016;126:1224-1232.

18 Liu Q, Rojas-Canales DM, Divito SJ, Shufesky WJ, Stolz DB, Erdos G, Sullivan ML, Gibson GA, Watkins SC, Larregina AT, Morelli AE: Donor dendritic cell-derived exosomes promote allograft-targeting immune response. J Clin Invest 2016;126:2805-2820.

19 Li XL, Li H, Zhang M, Xu H, Yue LT, Zhang XX, Wang S, Wang CC, Li YB, Dou YC, Duan RS: Exosomes derived from atorvastatin-modified bone marrow dendritic cells ameliorate experimental autoimmune myasthenia gravis by up-regulated levels of IDO/Treg and partly dependent on FasL/Fas pathway. J Neuroinflammation 2016;13:8.

-20 Wang L, Li N, Lin D, Zang Y: Curcumin protects against hepatic ischemia/reperfusion induced injury through inhibiting TLR4/NF-kappaB pathway. Oncotarget 2017;8:65414-65420.

-21 Liu Y, Ji H, Zhang Y, Shen X, Gao F, He X, Li GA, Busuttil RW, Kuchroo VK, Kupiec-Weglinski JW: Recipient T cell TIM-3 and hepatocyte galectin-9 signalling protects mouse liver transplants against ischemiareperfusion injury. J Hepatol 2015;62:563-572.

22 Lutz MB, Kukutsch N, Ogilvie AL, Rossner S, Koch F, Romani N, Schuler G: An advanced culture method for generating large quantities of highly pure dendritic cells from mouse bone marrow. J Immunol Methods 1999;223:77-92.

23 Czaya B, Singh S, Yanucil C, Schramm K, Faul C, Grabner A: Induction of an Inflammatory Response in Primary Hepatocyte Cultures from Mice. J Vis Exp 2017;121

-24 Chen Q Kong L, Xu X, Geng Q Tang W, Jiang W: Down-regulation of microRNA-146a in the early stage of liver ischemia-reperfusion injury. Transplant Proc 2013;45:492-496.

25 Thery C, Amigorena S, Raposo G, Clayton A: Isolation and characterization of exosomes from cell culture supernatants and biological fluids. Curr Protoc Cell Biol 2006; Chapter 3:Unit 322. 


\section{Cellular Physiology Cell Physiol Biochem 2018;46:740-756 and Biochemistry Published online:April 05, $2018 \quad$\begin{tabular}{l|l} 
DOI 2018 The Author(s). Published by S. Karger AG, Basel \\
www.karger.com/cpb
\end{tabular}}

Zheng et al.: Dexs Attenuate Liver Ischemia Reperfusion Injury by Modulating the Balance of Treg and Th17

-26 Dragovic RA, Gardiner C, Brooks AS, Tannetta DS, Ferguson DJ, Hole P, Carr B, Redman CW, Harris AL, Dobson PJ, Harrison P, Sargent IL: Sizing and phenotyping of cellular vesicles using Nanoparticle Tracking Analysis. Nanomedicine 2011;7:780-788.

27 Huijts CM, Santegoets SJ, Quiles Del Rey M, de Haas RR, Verheul HM, de Gruijl TD, van der Vliet HJ: Differential effects of inhibitors of the PI3K/mTOR pathway on the expansion and functionality of regulatory T cells. Clin Immunol 2016;168:47-54.

-28 Vicencio JM, Yellon DM, Sivaraman V, Das D, Boi-Doku C, Arjun S, Zheng Y, Riquelme JA, Kearney J, Sharma V, Multhoff G, Hall AR, Davidson SM: Plasma exosomes protect the myocardium from ischemia-reperfusion injury. J Am Coll Cardiol 2015;65:1525-1536.

29 Kim MG, Jung Cho E, Won Lee J, Sook Ko Y, Young Lee H, Jo SK, Cho WY, Kim HK: The heat-shock protein-70induced renoprotective effect is partially mediated by CD4+ CD25+ Foxp3 + regulatory T cells in ischemia/ reperfusion-induced acute kidney injury. Kidney Int 2014;85:62-71.

-30 Wachstein J, Tischer S, Figueiredo C, Limbourg A, Falk C, Immenschuh S, Blasczyk R, Eiz-Vesper B: HSP70 enhances immunosuppressive function of CD4(+)CD25(+)FoxP3(+) T regulatory cells and cytotoxicity in CD4(+)CD25(-) T cells. PLoS One 2012; 7:e51747.

31 Cao D, Wang M, Gong J, Wei S, Gong J, Li J: Exogenous vascular endothelial growth factor delivery prior to endothelial precursor cell transplantation in orthotopic liver transplantation-induced hepatic ischemia/ reperfusion injury. Liver Transpl 2017;23:804-812.

-32 Kolachala VL, Palle S, Shen M, Feng A, Shayakhmetov D, Gupta NA: Loss of L-selectin-guided CD8(+) but not $\mathrm{CD} 4(+)$, cells protects against ischemia reperfusion injury in a steatotic liver. Hepatology 2017;66:1258-1274.

-33 Sun L, Xu R, Sun X, Duan Y, Han Y, Zhao Y, Qian H, Zhu W, Xu W: Safety evaluation of exosomes derived from human umbilical cord mesenchymal stromal cell. Cytotherapy 2016;18:413-422.

34 Li F, Wang Y, Lin L, Wang J, Xiao H, Li J, Peng X, Dai H, Li L: Mast Cell-Derived Exosomes Promote Th2 Cell Differentiation via OX40L-OX40 Ligation. J Immunol Res 2016;2016:3623898.

35 Lu Z, Zuo B, Jing R, Gao X, Rao Q, Liu Z, Qi H, Guo H, Yin H: Dendritic cell-derived exosomes elicit tumor regression in autochthonous hepatocellular carcinoma mouse models. J Hepatol 2017;67:739-748.

36 Zanin-Zhorov A, Cohen IR: Signaling via TLR2 and TLR4 Directly Down-Regulates T Cell Effector Functions: The Regulatory Face of Danger Signals. Front Immunol 2013;4:211. 